\title{
Noise Robust Actuator Placement on Flexible Structures
}

\author{
Magnus Andersson \\ Department of Electrical Engineering \\ Linköping University, S-581 83 Linköping, Sweden \\ www: http://www. control.isy.liu.se \\ email: magnusa@isy.liu.se \\ 1997-09-01
}

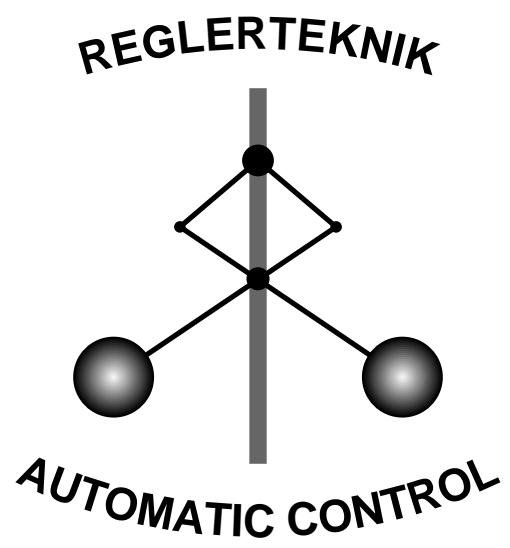

LINKÖPING

Technical reports from the Automatic Control group in Linköping are available as UNIX-compressed Postscript files by anonymous ftp at the address 130.236.20.24 (ftp.control.isy.liu.se). 


\title{
Noise Robust Actuator Placement on Flexible Structures
}

\author{
Magnus Andersson \\ Department of Electrical Engineering \\ Linköping University, 58183 Linköping, Sweden \\ e-mail: magnusa@isy.liu.se
}

\begin{abstract}
A novel criterion for placement of actuators on flexible mechanical structures is presented. Using simulated "measured modes" obtained from the model, the proposed criterion maximizes the correlation of the measured modes and the normal modes. The measured modes deviate from the normal modes due to damping, measurement noise and process noise. The statistical properties of the criterion are investigated. In simulations the computed actuator locations on a small aircraft-like model shows increased robustness properties against damping, for an acceptable loss of correlation. A computationally cheap actuator placement algorithm is proposed.
\end{abstract}

Key words: actuator placement, flexible structures, experimental modal analysis.

\section{Introduction}

The purpose of locating actuators (and sensors) on dynamical structures may be divided into three categories: experimental modal analysis [5], identification [6], and control [4]. Experimental modal analysis is the topic of analyzing the structure's modal properties using measurements of the modal parameters: resonance frequencies, mode shapes and damping.

Locating actuators on a flexible mechanical structure in order to measure its behavior to applied (sinusoidal) forces is common in, e.g., aerospace applications. The objective of the measuring is to validate the designed structure's dynamic properties in the low-frequency area. In order to predict the structure's behavior to externally applied forces, it is of vital importance to measure/estimate the mode shapes accurate. Therefore, locating actuators in order to excite the target modes of the structure is of high industrial interest.

Using an initial model of the structure, a design of the actuator placement can be made in advance of the real measuring. Usually a finite element (FE) model of medium to high complexity is used in a typical application. This means that the model may have 10,000 to 100,000 degrees of freedom (dof). In order to cope with the complexity of the model, a candidate set $\mathcal{A}_{c}$ of actuators must be specified for the actuator placement algorithms to become practical. Also, since the formulated optimization problems in general are nonconvex, sub-optimal algorithms must be used in order to fulfill the time and the memory requirements put on the actuator placement design. We will denote $\mathcal{A}_{r}$ as the outcome from the actuator placement algorithm.

Complex structures have often several near multiple modes (modes with close resonance frequencies). A criterion for locating actuators for the purpose of experimental modal analysis should therefore be able to excite the modes properly, without interference of near multiple modes.

\section{Basic Theory}

FE modeling of mechanical structures yields a mass matrix $M$, and a stiffness matrix $K$ of dimensions $l \times l$. The normal modes $\phi_{i}$ and resonance frequencies $\omega_{i}$ of the model are determined via the generalized eigenvalue problem

$$
\left(K-\omega_{i}^{2} M\right) \phi_{i}=0, \quad \phi_{i}^{T} M \phi_{i}=1, \quad i=\{1, \ldots, l\}
$$

where $l$ denotes the number of degrees of freedom (dof). The interpretation of the normal mode $\phi_{i}$ is as the shape of vibration (or relative amplitude) for the resonance frequency $\omega_{i}$.

If displacements are measured, the IO-behavior of a mechanical structure may be written

$$
\begin{aligned}
M \ddot{x}(t)+L \dot{x}(t)+K x(t) & =B u(t) \\
y(t) & =C x(t)
\end{aligned}
$$

where $x$ is the dof of the mechanical structure, $u$ is the vector of $m$ inputs, and $y$ the vector of $p$ outputs. In (2) $B$ (of dimension $l \times m$ ) describes the location of the actuators, and $C$ (of dimension $p \times l$ ) the location of the sensors. Notice: the damping matrix $L$ is in practice modeled separately from the FE modeling and is not further treated here, see [7].

The frequency response function (FRF) of (2) is defined as the transfer function $G(s)=Y(s) / U(s)$ evaluated at the imaginary axis, $s=j \omega$,

$$
G(j \omega)=C\left(K+j \omega L-\omega^{2} M\right)^{-1} B+D
$$


where $\omega$ denotes frequency. Defining $\Phi$ as

$$
\Phi=\left[\phi_{1}, \ldots, \phi_{l}\right]
$$

a transformation $x=\Phi q$, and a multiplication of $\Phi^{T}$ from the left on the upper equation in (2) yields:

$$
\begin{aligned}
\ddot{q}(t)+\bar{L} \dot{q}+\Omega q(t) & =\Phi^{T} B_{1} u(t) \\
y(t) & =C \Phi q(t)
\end{aligned}
$$

where

$$
\Omega=\operatorname{diag}\left(\omega_{1}^{2}, \ldots, \omega_{l}^{2}\right)
$$

and $\bar{L}=\Phi^{T} L \Phi$. If $\bar{L}$ is modeled diagonal,

$$
\bar{L}=\operatorname{diag}\left(2 \omega_{1} \xi_{1}, \ldots, 2 \omega_{l} \xi_{l}\right)
$$

the FRF of (5) can conveniently be written as

$$
G(j \omega)=\sum_{i=1}^{l} \frac{C \phi_{i} \phi_{i}^{T} B}{\omega_{i}^{2}-\omega^{2}+j 2 \xi_{i} \omega_{i} \omega}
$$

where $\xi_{i}$ is the damping ratio of mode $\phi_{i}$. The assumption of a diagonal $\bar{L}$ is valid in practice as well as in theory for flexible structures, see [3].

The Multivariate Mode Indicator Function (MMIF) in [8] is used in experimental modal analysis to indicate (multiple) modes of the structure. It is also used to determine the amplitudes and the phases (force patterns) of the sinusoidal input forces. The force patterns are computed in order to excite the structures for accurate mode estimations. This technique is called normal mode force appropriation (or phase resonance testing). Normal mode force appropriation is today a well-established technique for exciting a single mode of a structure normally using multiple actuators.

The frequency response output $\bar{Y}$ is defined as

$$
\bar{Y}_{i}=G \bar{U}_{i}, \quad \text { for } \quad u=\bar{U}_{i} \sin \left(\omega_{i} t\right)
$$

If a single mode $\phi_{i}$ is "purely" excited (at $\omega=\omega_{i}$ ), will $\bar{Y}_{i}$ in (8) equal $-j C \phi_{i} \phi_{i}^{T} B \bar{U}_{i} / 2 \xi_{i} \omega_{i}^{2}$. Dropping the notation of $C$, an estimate $\hat{\phi}_{i}^{d}$ of $\phi_{i}$ may be defined as

$$
\hat{\phi}_{i}^{d}=\operatorname{Im}\left\{\bar{Y}_{i}\right\}
$$

for an appropriate chosen force pattern $\bar{U}_{i}$. In (10), the superscript $d$ denotes damping.

If a single mode $\phi_{i}$ is to be excited at a resonance frequency $\omega_{i}$, the real part of $\bar{Y}_{i}$ in (9) should vanish, due to (10). The objective is therefore to find the force pattern $\bar{U}_{i}$ minimizing the real part of $\bar{Y}_{i}$. This objective corresponds to the solution of the minimization problem

$$
\lambda_{i, \min }=\min _{\left\|U_{i}\right\|=1} \frac{\left\|\operatorname{Re}\left\{\bar{Y}_{i}\right\}\right\|^{2}}{\left\|\bar{Y}_{i}\right\|^{2}}
$$

where $\|\bar{Y}\|^{2}=\bar{Y}^{H} \bar{Y}$, and $\bar{Y}^{H}$ is the complex conjugate of $\bar{Y}$.

It follows that $\lambda_{i, \min }$ in (11) is the smallest eigenvalue $\lambda_{i, k}$ of the generalized eigenvalue problem

$$
\left(A_{i}-\lambda_{i, k}\left(A_{i}+B_{i}\right)\right) U_{i, k}=0, \quad k=1, \ldots, m
$$

where $A_{i}=\operatorname{Re}\left\{\bar{Y}_{i}\right\}^{T} \operatorname{Re}\left\{\bar{Y}_{i}\right\}$ and $B_{i}=\operatorname{Im}\left\{\bar{Y}_{i}\right\}^{T} \operatorname{Im}\left\{\bar{Y}_{i}\right\}$. The eigenvalue problem in (12) is of small dimension, $m \times m$, if $m$ actuators are used. The set of $m$ eigenvalues $\lambda_{i, k}$ in (12) can for each $i$ be used to detect the single mode $\phi_{i}$ if $\lambda_{i, 1}=\lambda_{i \text {, min }} \approx 0$, two near multiple modes if also $\lambda_{i, 2} \approx 0$, etc. Moreover, the eigenvectors $U_{i, k}$ in (12) are candidates as input force patterns $\bar{U}_{i}$ to the structure.

\section{Noise Model}

Assuming the entries of the measurement noise vector $\phi_{i}^{m n}$ are unbiased and independent between the sensors, it can be modeled as a normally distributed random vector of zero-mean, i.e.,

$$
\begin{aligned}
& \hat{\phi}_{i}^{m n}=\left[\ldots, x_{i, j}^{m n}, \ldots\right], \quad x_{i, j}^{m n} \sim \mathrm{N}\left(0, \sigma_{i}^{m n}\right) \\
& x_{i, j_{1}}^{m n}, x_{j, j_{2}}^{m n} \text { independent } \quad \forall i, \forall j, \forall j_{1} \neq j_{2}
\end{aligned}
$$

where the noise variance equals $\left(\sigma_{i}^{m n}\right)^{2}$.

Moreover, if the locations of the process noise sources are known, the frequency response function $G^{p n}$ from the $N_{p n}$ process noise sources to the $p$ sensors, can be computed. Using $G^{p n}$ together with a process noise source model, we arrive at the process noise model

$$
\begin{gathered}
\hat{\phi}_{i}^{p n}=\sum_{k=1}^{N_{p n}} \hat{\phi}_{i, k}^{p n}=\sum_{k=1}^{N_{p n}} \phi_{i, k}^{p n} x_{i, k}^{p n}, \quad x_{i, k}^{p n} \sim \mathrm{N}\left(0, \sigma_{i, k}^{p n}\right) \\
x_{i, k_{1}}^{p n}, x_{j, k_{2}}^{p n} \quad \text { independent } \quad \forall i, \forall j, \forall k_{1} \neq k_{2}
\end{gathered}
$$

where the process noise sources are assumed to be unbiased and independent. We also assume the measurement noise and the process noise to be independent,

$$
x_{i, j}^{m n}, x_{k, l}^{p n} \quad \text { independent } \quad \forall i, \forall j, \forall k, \forall l
$$

The choice of noise variance levels may be seen as a design variable and has to be chosen properly. One approach is to set the measurement noise levels $\left(\sigma_{i}^{m n}\right)^{2}$ and the process noise levels $\left(\sigma_{i, k}^{p n}\right)^{2}$ as a fraction of a (simulated) measured response. 
For measurement noise variance: define, $\overline{\mathcal{E}}_{i}$ as the mean "power" measured by the sensors,

$$
\begin{aligned}
\overline{\mathcal{E}}_{i}\left(\mathcal{A}_{t}, \mathcal{S}_{t}\right) & =\frac{1}{\left|\mathcal{A}_{t}\right|} \sum_{k=1}^{\left|\mathcal{A}_{t}\right|} \mathcal{E}_{i, k}, \quad \text { where } \\
\mathcal{E}_{i, k}\left(a_{k}, \mathcal{S}_{t}\right) & =\frac{\left|\hat{\phi}_{i}^{d}\right|^{2}}{\left|\mathcal{S}_{t}\right|}, \quad a_{k} \in \mathcal{A}_{t}
\end{aligned}
$$

where $\left|\hat{\phi}_{i}^{d}\right|^{2}$ is the measured "power" for a single actuator $a_{k}$ in a specified test set $\mathcal{A}_{t}$ of actuators, and for a test set $\mathcal{S}_{t}$ of sensors.

For process noise is the noise variance level design more simple. One approach is to let the process noise variance be a fraction of the level of the commanded input force $|U|^{2}$.

\section{A Noise Robust Actuator Placement Criterion}

The estimated mode $\hat{\phi}_{i}^{n d}$ from the structure, may be modeled

$$
\hat{\phi}_{i}^{n d}=\hat{\phi}_{i}^{d}+\hat{\phi}_{i}^{m n}+\hat{\phi}_{i}^{p n}
$$

where superscript $d$ denotes damping, $m n$ measurement noise, and $p n$ process noise.

A generalization of the modal assurance criterion (MAC) in [1], may be defined as

$$
\operatorname{ndMAC}_{i}=\frac{\mathrm{E}\left[\left|\phi_{i}^{T} \hat{\phi}_{i}^{n d}\right|^{2}\right]}{\mathrm{E}\left[\left|\phi_{i}\right|^{2}\left|\hat{\phi}_{i}^{n d}\right|^{2}\right]}
$$

where E denotes expectation.

It can be verified that $\mathrm{ndMAC}_{i}$ in (18) equals:

$\operatorname{ndMAC}_{i}=\frac{\left|\phi_{i}^{T} \hat{\phi}_{i}^{d}\right|^{2}+\left|\phi_{i}\right|^{2}\left(\sigma_{i}^{m n}\right)^{2}+\sum_{k}\left|\phi_{i}^{T} \phi_{i, k}^{p n}\right|^{2}\left(\sigma_{i, k}^{p n}\right)^{2}}{\left|\phi_{i}\right|^{2}\left|\hat{\phi}_{i}^{d}\right|^{2}+p\left|\phi_{i}\right|^{2}\left(\sigma_{i}^{m n}\right)^{2}+\left|\phi_{i}\right|^{2} \sum_{k}\left|\phi_{i, k}^{p n}\right|^{2}\left(\sigma_{i, k}^{p n}\right)^{2}}$

for the proposed noise model in (13)-(15), and (17), see Appendix A, where also other important properties of the criterion are shown.

A noise-robust actuator placement criterion may be formulated:

$$
\mathcal{A}_{r}=\underset{\substack{\mathcal{A}_{r} \subset \mathcal{A}_{c} \\\left|\mathcal{A}_{r}\right|=m}}{\arg \max }|W \mathcal{N}|_{q}
$$

where $W$ is a diagonal weight matrix, $1 \leq q \leq \infty$, and $\mathcal{N}$ the vector

$$
\mathcal{N}=\left[\ldots,\left(\operatorname{ndMAC}_{i}\right)^{\frac{1}{2}}, \ldots\right]^{T}, \quad i=1, \ldots, N_{t m}
$$

The $\mathrm{dMAC}_{i}$ is defined as the $\mathrm{ndMAC}_{i}$ for zero noise levels, i.e.,

$$
\mathrm{dMAC}_{i}=\frac{\left|\phi_{i}^{T} \hat{\phi}_{i}^{d}\right|^{2}}{\left|\phi_{i}\right|^{2}\left|\hat{\phi}_{i}^{d}\right|^{2}}
$$

Notice that the criterion in [5] equals (18), (20), and (21) with $\sigma_{i}^{m n}=0, \sigma_{i}^{p n}=0, W=I$ (identity matrix) and $q=2$, i.e., with no noise model.

\section{Actuator Placement Algorithm}

Recall that a full search for $\mathcal{A}_{r}$ among $\mathcal{A}_{c}$ is often not feasible due to the computational complexity of a criterion $P$, and the size of the model. Instead sub-optimal algorithms computing $\mathcal{A}_{r}$ must be used. Here an algorithm with found minor sub-optimality is proposed.

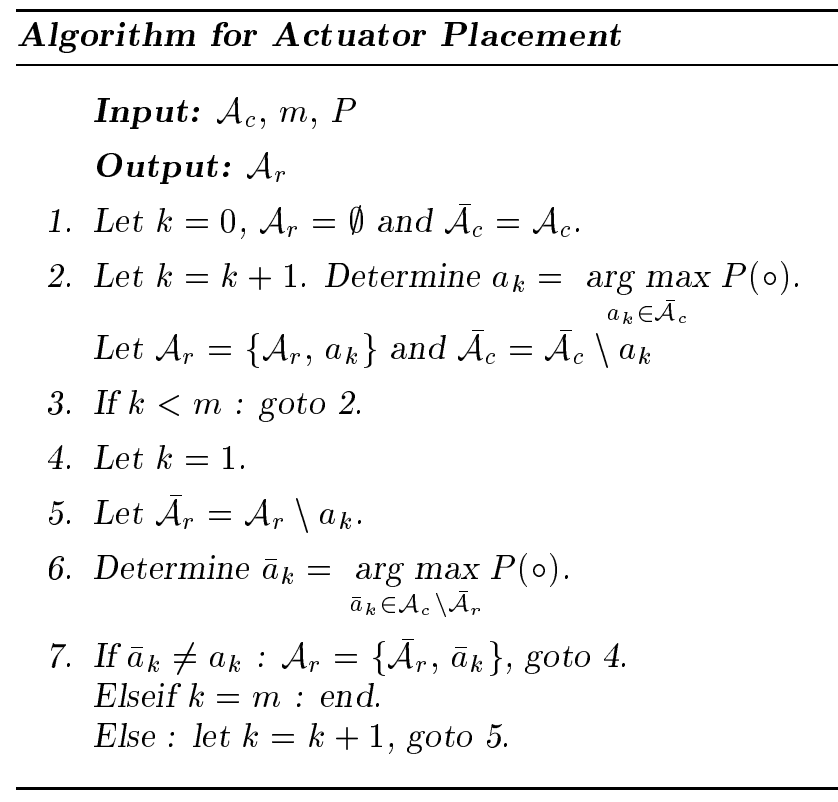

In [2], the termination of the proposed algorithm is shown.

\section{Example}

The measurement noise levels $\left\{\sigma_{0}^{m n}, \sigma_{l}^{m n}, \sigma_{h}^{m n}\right\}$ (zero, low, and high) are here related to $\overline{\mathcal{E}}_{i}$ as,

$$
\begin{aligned}
\sigma_{0}^{m n} & \Leftrightarrow \frac{\overline{\mathcal{E}}_{i}}{\left(\sigma_{i}^{m n}\right)^{2}}=0 \quad \leftrightarrow \infty \mathrm{dB} \mathrm{SNR} \\
\sigma_{l}^{m n} & \Leftrightarrow \frac{\overline{\mathcal{E}}_{i}}{\left(\sigma_{i}^{m n}\right)^{2}}=10^{4} \leftrightarrow 40 \mathrm{~dB} \mathrm{SNR} \\
\sigma_{h}^{m n} & \Leftrightarrow \frac{\overline{\mathcal{E}}_{i}}{\left(\sigma_{i}^{m n}\right)^{2}}=10^{2} \leftrightarrow 20 \mathrm{~dB} \mathrm{SNR}
\end{aligned}
$$


for

$$
\mathcal{A}_{t}=\mathcal{S}_{t}=\{\text { all transl. dof }\}
$$

In Fig. 1 an aircraft like model is visualized. The model was constructed to have several near multiple modes, where denotes $\circ$ a grid point representing 3 translational and 3 rotational dof.

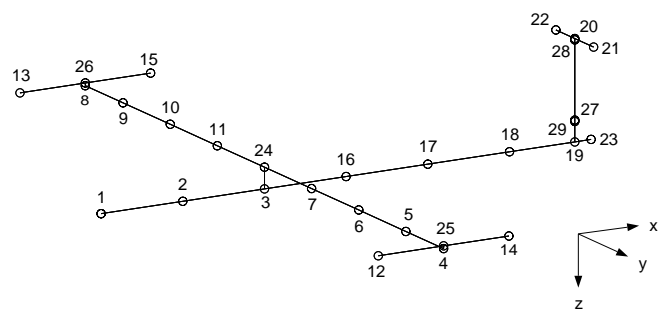

Figure 1: The geometry of the model for actuator placement design. The grids are denoted $\circ$.

Using the proposed actuator placement algorithm, the criterion $P=|\mathcal{N}|_{1}$ the measurement noise levels defined in (23), zero process noise variance level, and the candidate set

$$
\mathcal{A}_{c}=\{\text { all transl. dof }\}
$$

of actuators, the resulting sets of three actuator locations $\mathcal{A}_{r}$ become as in Table 1 . The ndMAC $_{i}$-values are decreasing with the increase of the measurement noise level. It is seen that for a higher level of noise variance, the actuators are moved towards the end of the wings and the tail. The subscripts $x, y, z$ denotes the direction of the applied force.

The actuator locations in Table 1 are henceforth defined as in Table 2.

In Table 3 , the $\mathrm{dMAC}_{i}$-values for the actuator locations is presented. Notice the small loss in the $\mathrm{dMAC}_{i}$ for an increasing level of noise variance.

A robustness test against the level of measurement noise levels is presented in Table 4 . It is readily seen that an actuator placement design based on a low measurement noise variance has bad robustness properties against a higher levels of measurement noise.

From an engineering point of view, one may argue that the higher the noise variance levels are, the better the Signal-to-Noise-Ratio will be. Since, when the

Table 1: The resulting actuator locations $\mathcal{A}_{r}$, and the $\mathrm{ndMAC}_{i}$-values for the nine low-frequency modes.

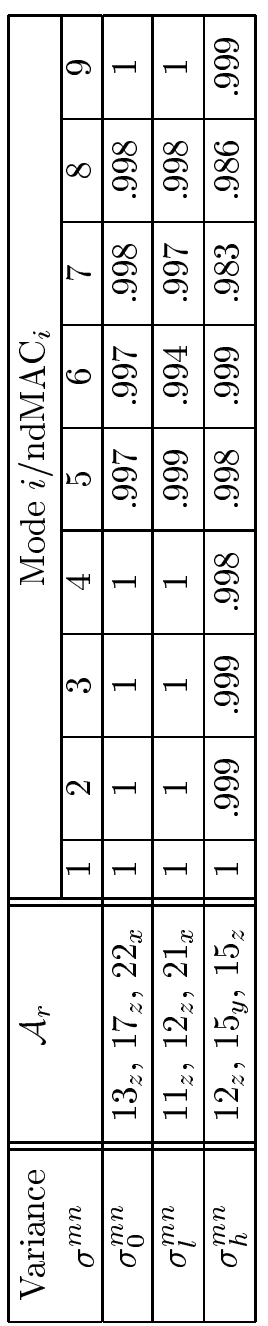

Table 2: The defined actuator locations.

\begin{tabular}{|l|l|}
\hline $\mathcal{A}_{r}^{0}$ & $13_{z}, 17_{z}, 22_{x}$ \\
\hline $\mathcal{A}_{r}^{l}$ & $11_{z}, 12_{z}, 21_{x}$ \\
\hline $\mathcal{A}_{r}^{h}$ & $12_{z}, 15_{y}, 15_{z}$ \\
\hline
\end{tabular}


Table 3: The resulting actuator locations $\mathcal{A}_{r}$, and the $\mathrm{dMAC}_{i}$-values for the nine low-frequency modes.

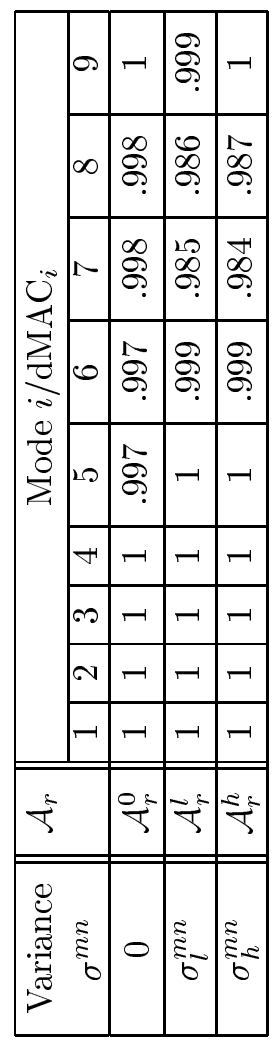

Table 4: Robustness properties of the actuator locations against different levels of measurement noise.

\begin{tabular}{|c|c|c|c|}
\hline Robustness Criterion & $\mathcal{A}_{r}^{0}$ & $\mathcal{A}_{r}^{l}$ & $\mathcal{A}_{r}^{h}$ \\
\hline Mean $\left(\right.$ ndMAC $\left._{i}\right), \sigma_{l}^{m n}$ & .997 & .998 & .996 \\
\hline Mean $\left(\right.$ ndMAC $\left._{i}\right), \sigma_{h}^{m n}$ & .869 & .962 & .989 \\
\hline Min $\left(\operatorname{ndMAC}_{i}\right), \sigma_{l}^{m n}$ & .996 & .993 & .984 \\
\hline Min $\left(\operatorname{ndMAC}_{i}\right), \sigma_{h}^{m n}$ & .762 & .883 & .981 \\
\hline
\end{tabular}

noise variance levels are increased, the actuators are placed such that the structure is more easily excited in order to yield good mode estimates. That is, for a fixed level of input force from the actuators, the amplitude of the measured displacements increases with the noise variance levels. That is why the actuators are placed farther away from the center of gravity when the noise variance levels are increased. Notice that the farther away from the center of gravity the actuators are placed, the less good the mode estimates will be for zero noise variance level. However, the price paid for this robustness against noise is low since the mode estimates are "good enough" when the actuators are placed with a high level of designed noise variance. In Table 5 the SNR for the noise level corresponding to $\sigma_{l}^{m n}$ is presented. It is clearly seen that the SNR is increased with the designed noise variance levels.

Table 5: The Signal-to-Noise-Ratio for a fixed level of noise variance level.

\begin{tabular}{|c|c|c|}
\hline $\mathcal{A}_{r}^{0}$ & $\mathcal{A}_{r}^{l}$ & $\mathcal{A}_{r}^{h}$ \\
\hline$-16.2 \mathrm{~dB}$ & $0 \mathrm{~dB}$ & $10.5 \mathrm{~dB}$ \\
\hline
\end{tabular}

\section{Conclusions}

A novel criterion for actuator placement for the purpose of experimental modal analysis is presented. The statistical properties of the criterion is good under some easily fulfilled additional conditions. Increased robustness properties are obtained for a minor loss of correlation. The proposed, computationally cheap actuator placement algorithm is in simulations found to yield actuator locations with good properties. In a multiple-mode aircraft-like model example the actuator locations have the desired properties for experimental modal analysis.

\section{References}

[1] R. Allemang and D. Brown. A correlation coefficient for modal vector analysis. In Proc. 1st International Modal Analysis Conference, pages 110-116, 1982.

[2] M. Andersson. Experimental design and updating of finite element models. Lic. thesis, Automatic Control, Dept. of EE, Linköping University, 58183 Linköping, Sweden, April 1997.

[3] M. Géradin and D. Rixen. Mechanical Vibrations - Theory and Application to Structural Dynamics. Wiley, 1994.

[4] X. Guangqian and P. M. Bainum. Actuator placement using degree of controllability for discretetime systems. Trans. of the ASME, 114:508-516, September 1992. 
[5] P. S. Holmes, J. R. Wright, and J. E. Cooper. Optimum exciter placement for normal mode force appropriation using an a priori model. In Proc. 14th International Modal Conference, 1996.

[6] P. C. Shah and F. E. Udwadia. A methodology for optimal sensor locations for identification of dynamic systems. Journal of Applied Mechanics, 45:188196, March 1978.

[7] M. Soni. Finite element procedure in damping design. In Proceedings of the 14th Inernational Seminar on Modal Analysis, pages 8.1.1-8.1.48, Leuven, 1989.

[8] R. Williams, J. Crowley, and H. Vold. The multivariate mode indicator function in modal analysis. In Proceedings of the 3rd International Modal Analysis Conference, pages 66-70, 1985.

\section{A Properties of the ndMAC $i$}

Proof of (19):

Evaluating (18), ndMAC $_{i}$ equals

$$
\operatorname{ndMAC}_{i}=\frac{\mathrm{E}\left[\left|\phi_{i}^{T} \hat{\phi}_{i}^{d}\right|^{2}+\left|\phi_{i}^{T} \hat{\phi}_{i}^{m n}\right|^{2}+\left|\sum_{k} \phi_{i}^{T} \hat{\phi}_{i, k}^{p n}\right|^{2}\right]}{\mathrm{E}\left[\left|\phi_{i}\right|^{2}\left|\hat{\phi}_{i}^{d}\right|^{2}+\left|\phi_{i}\right|^{2}\left|\hat{\phi}_{i}^{m n}\right|^{2}+\left|\phi_{i}\right|^{2}\left|\sum_{k} \hat{\phi}_{i, k}^{p n}\right|^{2}\right]}
$$

due to the independence properties of $x_{i, j}^{m n}$ and $x_{k, l}^{p n}$, see (13)-(15). For instance,

$$
\begin{aligned}
\mathrm{E}\left[\left|\sum_{k} \phi_{i}^{T} \hat{\phi}_{i, k}^{p n}\right|^{2}\right] & =\mathrm{E}\left[\sum_{k} \sum_{l}\left(\phi_{i}^{T} \hat{\phi}_{i, k}^{p n}\right)\left(\phi_{i}^{T} \hat{\phi}_{i, l}^{p n}\right) x_{i, k}^{p n} x_{i, l}^{p n}\right] \\
& =\sum_{k}\left|\phi^{T} \hat{\phi}_{i, k}^{p n}\right|^{2}\left(\sigma_{i, k}^{p n}\right)^{2}
\end{aligned}
$$

noticing that $\sum_{k} \phi_{i}^{T} \hat{\phi}_{i, k}^{p n}$ is a scalar, and

$$
\mathrm{E}\left[\left|\phi_{i}\right|^{2}\left|\hat{\phi}_{i}^{m n}\right|^{2}\right]=\mathrm{E}\left[\left|\phi_{i}\right|^{2} \sum_{l}\left(x_{i, l}^{m n}\right)^{2}\right]=p\left|\phi_{i}\right|^{2}\left(\sigma_{i}^{m n}\right)^{2}
$$

since there are $p$ terms in the sum in (26).

The ndMAC $i$ must fulfill some criterions in order to be valid for actuator placement purposes:

$$
\begin{array}{ll}
\text { C1: } & \operatorname{ndMAC}_{i} \leq 1 \\
\text { C2a: } & \frac{\partial \operatorname{ndMAC}_{i}}{\partial\left(\sigma_{i}^{m n}\right)^{2}}<0, \forall i \\
\text { C2b: } & \frac{\partial \operatorname{ndMAC}_{i}}{\partial\left(\sigma_{i, k}^{p n}\right)^{2}}<0, \forall i, k \\
\text { C3a: } \lim _{\left(\sigma_{i}^{m n}\right)^{2} \rightarrow \infty} \operatorname{ndMAC}_{i}=\epsilon_{i}^{m n} \ll 1, \forall i \\
\text { C3b: } \lim _{\substack{\left.\sigma_{i, n}^{m}\right)^{2} \rightarrow \infty \\
\sigma_{i, k}}} \operatorname{ndMAC}_{i}=\epsilon_{i, k}^{p n} \ll 1, \forall i, k \\
\text { C4: } \begin{array}{c}
\left.\lim _{i}^{m n}\right)^{2} \rightarrow 0 \\
\left(\sigma_{i, k}^{p n}\right)^{2} \rightarrow 0, \forall k
\end{array} & \operatorname{ndMAC}_{i}=\operatorname{dMAC}_{i}, \forall i
\end{array}
$$

The formulated criterions in (27) can be motivated intuitively:

C1: The ndMAC ${ }_{i}$-values should be less than 1 .

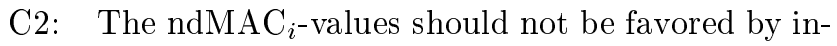
creased noise levels.

C3: For high noise levels, the ndMAC ${ }_{i}$-values should be low.

C4: Low noise levels should not significantly affect the $\operatorname{ndMAC}_{i}$-values.

The criterions $\mathrm{C} 1$, and $\mathrm{C} 4$ is easily verified. The criterions $\mathrm{C} 2 \mathrm{a}, \mathrm{C} 2 \mathrm{~b}, \mathrm{C} 3 \mathrm{a}$, and $\mathrm{C} 3 \mathrm{~b}$ can be verified under some additional conditions:

C2a: If

$$
\begin{aligned}
\frac{\left|\phi_{i}^{T} \hat{\phi}_{i}^{d}\right|^{2}}{\left|\phi_{i}\right|^{2}\left|\hat{\phi}_{i}^{d}\right|^{2}}> & \frac{1}{p}\left(1+\frac{\sum_{k}\left|\phi_{i, k}^{p n}\right|^{2}\left(\sigma_{i, k}^{p n}\right)^{2}}{\left|\hat{\phi}_{i}^{d}\right|^{2}}\right) \\
& -\frac{\sum_{k}\left|\phi_{i}^{T} \phi_{i, k}^{p n}\right|^{2}\left(\sigma_{i, k}^{p n}\right)^{2}}{\left|\phi_{i}\right|^{2}\left|\hat{\phi}_{i}^{d}\right|^{2}}
\end{aligned}
$$

the $\mathrm{ndMAC}_{i}$ is monotonically decreasing in $\sigma_{i}^{m n}$.

\section{C2b: If}

$$
\begin{aligned}
\frac{\left|\phi_{i}^{T} \hat{\phi}_{i}^{d}\right|^{2}}{\left|\phi_{i}\right|^{2}\left|\hat{\phi}_{i}^{d}\right|^{2}}>\frac{\left|\phi_{i}^{T} \phi_{i, l}^{p n}\right|^{2}}{\left|\phi_{i}\right|^{2}\left|\phi_{i, l}^{p n}\right|^{2}}( & 1+\frac{p\left(\sigma_{i}^{m n}\right)^{2}}{\left|\hat{\phi}_{i}^{d}\right|^{2}} \\
& \left.+\frac{\sum_{k}\left|\phi_{i, k}^{p n}\right|^{2}\left(\sigma_{i, k}^{p n}\right)^{2}}{\left|\hat{\phi}_{i}^{d}\right|^{2}}\right)^{(29)}
\end{aligned}
$$

then $\mathrm{ndMAC}_{i}$ is monotonically decreasing in $\sigma_{i, l}^{p n}$.

C3a,b: the ndMAC $i$ asymptotically converge to:

$$
\begin{aligned}
\lim _{\left(\sigma_{i}^{m n}\right)^{2} \rightarrow \infty} \operatorname{ndMAC}_{i} & =\frac{1}{p}, \quad \forall i \\
\lim _{\left(\sigma_{i, k}^{p n}\right)^{2} \rightarrow \infty} \operatorname{ndMAC}_{i} & =\frac{\left|\phi_{i}^{T} \phi_{i, k}^{p n}\right|^{2}}{\left|\phi_{i}\right|^{2}\left|\phi_{i, k}^{p n}\right|^{2}}, \quad \forall i, \forall k
\end{aligned}
$$

compare with (19).

Proofs of C1-C4 can be found in [2]. 